\title{
Calorimetrische Untersuchungen
}

von

\section{F. Stohmann. \\ Neunzehnte Abhandlung. \\ Ueber den Wärmewerth der Säuren der Oxalsäure-Reihe und der Fumar- und Maleïnsänre \\ Fon}

\section{F. Stohmann, Cl. Kleber und H. Langbein.}

Zweck dieser bereits vor zwei Jahren begonnenen Arbeit war zunächst, zu erforschen, wie weit die zahlreichen Isomerien, welche bei den sogenannten Adipinsäuren $\mathrm{C}_{6} \mathrm{H}_{10} \mathrm{O}_{4}$ auftreten, einen Einfluss auf deren Wärmewerth ausüben. Sodann musste uns erwünscht sein, unsere alten, durch Verbrennung mit Kaliumchlorat gewonnenen Zahlen zu controlliren, und endlich haben sich, bei von Louguinine ${ }^{1}$ ) ausgeführten Verbrennungen einer Anzahl hierher gehörender Säuren, eigenthümliche Verhältnisse unter den Homologen gezeigt, deren nähere Ergründung erwünscht erschien.

\section{Oxalsäure $\left\{\begin{array}{l}\mathrm{COOH} \\ \mathrm{COOH}\end{array}\right.$ oder $\mathrm{C}_{2} \mathrm{H}_{2} \mathrm{O}_{4} .90$.}

Zur Darstellung der reinen Säure wurde die krystallisirte Säure bei einer Temperatur von $60^{\circ}-70^{\circ}$, in dünnen Schichten auf Tellern ausgebreitet, der Verwitterung überlassen und die nicht geschmolzene Masse zunächst bei $100^{\circ}$ völlig entwässert. Die wasserfreie Säure wurde dann in einem kupfernen Rohre, welches in einem Paraffinbade constant auf einer Temperatur von $157^{\circ}$ erhalten wurde, unter beständigem Darüberleiten eines Luftstromes sublimirt. Bei Innehaltung der angegebenen Temperatur gelingt die Sublimation der Säure so leicht, dass grosse Mengen derselben ohne jeden Umstand darzustellen sind.

Die Verbremnung der Oxalsäure in der Bombe bietet ganz besondere Schwierigkeiten. Sie setzt der Entzündung dieselben

\footnotetext{
1) Compt. rend. 107, 597 .
} 
Hindernisse entgegen, wie die Mellithsäure, (s. Abh. XVIII. $\left.{ }^{1}\right)$ ), nur noch in erheblich höherem Grade. Während die EntHlammung der Mellithsäure durch Zusatz geringer Mengen von Stearinsäure zu erreichen war, so war hierzu bei der Oxalsäure eine viel grössere Menge erforderlich. Als z. B. eine Pastille von 1,9819 Grm. Oxalsäure mit 0,1981 Grm. Stearinsäure zu entzünden versucht wurde, verbrannte nur die oberste Schicht bis zu einer ganz geringen Tiefe; dasselbe war der Fall bei Pastillen von 1,9608 Grm., resp. 2,6363 Grm. Oxalsäure, denen 0,4561 und $0,4958 \mathrm{Grm}$. Stearinsäure zugefügt. war, obgleich die Wärmemenge, welche die leicht verbrennende Stearinsäure hätte liefern können, in den beiden letzten Fällen etwa $2^{1 / 2}$ mal so hoch war als die Menge der Wärme, welche bei der Verbreınung der Oxalsäure zu erwarteı gewesen sein würde. Wir erreichten unsern $Z_{\text {weck }}$ erst, als noch grössere Mengelı von Stearinsäure angewandt wurden, wobei dann freilich die durch die Verbrennung der Oxalsäure producirte Wärmemenge immer nur einen Bruchtheil von der Wärmemenge ausmachte, welche vou der Stearinsäure geliefert wurde. Um hierdurch etwa entstehende Fehler klar zu Tage treten zu lassen, haben wir bei den einzelneı Verbrennungen einerseits die Mengen der Oxalsäure und andererseits das Verhältniss der Oxalsäure zur Stearinsäure innerhalb möglichst weiter Grenzen wechselnd genommen. Die sich aus den nachfolgenden Zahlen ergebende, über alles Erwarten hinausgehende Uebereinstimmung der Resultate der unter den verschiedensten Bedingungen angestellten Beobachtungen ergiebt aber die $\mathrm{Zu}$ lässigkeit des angewandten Verfahrens. Die aus den Resultaten abgeleitete Werthzahl der Oxalsäure könnte demnach nur dann fehlerhaft sein, wenn der Wärmewerth der von uns benutzten Stearinsäure nicht richtig bestimmt wäre. Da aber die von uns für die Oxalsäure ermittelte Zahl mit der von anderen Beobachtern gefundenen in vollem Einklange steht, so ist damit auch zugleich eine Bestätigung für die Richtigkeit des Werthes der Stearinsäure gegeben.

$\mathrm{Zu}$ dieser Schwierigkeit, welche die Verbrennung der Oxalsäure bietet, kam noch eine weitere hinzu. Unter drei

1) Dies. Journ. $\{2\rceil 40,141$. 
204 Stohmann, Kleber u. Langbein: Ueber den

von vier, sonst glücklich verlaufenen Verbrennungen fand sich auf dem Boden des Schälchens eine geringe Menge von unverbrannter Kohle, im Betrage von 0,0008 Grm., 0,0006 Grm. und $0,0025 \mathrm{Grm}$. Der Wärmewerth derselben ist den Correctionen beigefügt und zwar ist dafür die von Berthelot ${ }^{1}$ ) gefundene Zahl 8137,4 cal. für $1 \mathrm{Grm}$. amorphen Kohlenstoff angenommen.

Die Beobachtungen ergaben folgende Werthe:

\begin{tabular}{|c|c|c|c|c|c|}
\hline & & 1 & 2 & 3 & 4 \\
\hline Substanz: Oxalsäure & Grm. & 1,6961 & 2,6409 & 2,6225 & 4,4193 \\
\hline Stearins. & Grm. & 0,6725 & 0,7176 & 0,7573 & 0,9225 \\
\hline$v$ & pp. & $+0,72$ & $+0,66$ & $+0,72$ & $+0,76$ \\
\hline$v^{2}$ & pp. & $-0,22$ & $-0,26$ & $-0,26$ & $-0,59$ \\
\hline $\boldsymbol{t}$ & pp. & 299,3 & 294,7 & 289,1 & 292,0 \\
\hline $\boldsymbol{t}^{\prime}$ & pp. & 520,3 & 545,4 & 550,4 & 631,2 \\
\hline$\sum_{I}^{n-1} \nsucc r$ & pp. & 1870 & 1946 & 1956 & 2212 \\
\hline$n$ & & 5 & $\check{\jmath}$ & 5 & 5 \\
\hline$\frac{\vartheta_{n}+\vartheta_{1}}{2}$ & pp. & 411 & 421 & 421 & 463 \\
\hline$\Sigma \Delta t$ & pp. & $+0,45$ & $+0,6$ & $+0,6$ & $+1,8$ \\
\hline$\Psi_{n}$ (corr.) & Grad & 19,5434 & 19,8960 & 19,9653 & 21,1133 \\
\hline$\vartheta_{1}$ & Grad & 16,5516 & 16,4877 & 16,4130 & 16,4538 \\
\hline$\left(\vartheta_{n}-\vartheta_{1}\right) 2500$ & cal. & 7479,5 & 8520,8 & 8880,8 & 11648,8 \\
\hline Correct. f. Eisen & cal. & $-9,1$ & $-9,1$ & $-9,1$ & $-9,1$ \\
\hline " f. $\mathrm{HNO}_{3}$ & eal. & $-9,0$ & $-6,0$ & -6.1 & $-5,1$ \\
\hline f. Stearins. ${ }^{2}$ & ) cal. & $-6304,1$ & $-6726,9$ & $-7099,0$ & $-8647,6$ \\
\hline f. Kohle & cal. & 0 & $+6,5$ & $+4,9$ & $+20,3$ \\
\hline
\end{tabular}

Wärmewerth der Oxalsäure:

pro Grm. pro Grm.-Mol.

cal. Cal.

1. 682,3 . . . 61,4

2. 676,0 . . . 60,8

3. 675,5 . . . 60,8

4. 680,4 . . . 61,2

Mittel 678,6 . . 61,1 für constantes Volum

60,2 für constanten Druck

Bildungswärme 196,8 Cal.

1) Compt. rend. 108, 1144.

2) 1 Grm. Stearinsiaure $=9374,1$ cal. (vergl. Abh. XVIII). 
Wärmewerth der Säuren der 0xalsäure-Reihe etc. $20 ذ$

Andere Forscher haben folgende Werthe für die Oxalsäure gefunden:

Berthelot ${ }^{1}$ ): Verbrennungswärme $60,2 \mathrm{Cal}$.

Thomsen $\left.{ }^{2}\right)$ : Bildungswärme 202,5 Cal. Verbrennungswärme $59,8 \mathrm{Cal}^{3}$ )

Louguinine ${ }^{4}$ ): Verbrennungswärme 63,4 Cal., abgeleitet aus der Verbrennungswärme des Oxalsäure - Diäthyläthers. Merkwürdiger Weise nimmt Louguinine, ohne neue Bestimmungen gemacht zu haben, in einer späteren Arbeit ${ }^{5}$ ) nicht diese Zahl, sondern $60 \mathrm{Cal}$. als Verbrennungswärme der Oxalsäure an.

$\mathrm{Jahn}^{6}$ ): Verbrennungswärme 60,5 Cal.

Hiernach ist unsere frühere mit Kaliumchlurat ausgeführte Bestimmung des Wärmewerthes der Oxalsäure, welche 51,4 Cal. ergab, (Abh. $\left.I^{7}\right)$ ) als zu niedrig zu betrachten. ${ }^{8}$ )

1) Ann. Chim. [5] j, 305.

2) Thermochem. Untersuchungen 2, 294.

3) Berechnet nach den von Th om sen benutzten Zablen $\mathrm{C}=96960 \mathrm{cal}$., $\mathrm{H}_{2}=68360$ cal.

4) Ann. Chim. [6] 8, 140.

5) Compt. rend. 107, 598.

6) Ann. Phys. [2] 37, 408.

7) Dies. Journ. [2] 31, 301.

8) Um nicht alten Streit von neuem wieder zu entfachen, enthalte ich mich, auf den Schlusssatz, welchen $\mathrm{Hr}$. Jahn seiner Arbeit beigefügt hat, näher einzugehen und kann dieses um so eher, als weder von mir, noch meines Wissens von irgend einem Anderen, die Resultate der Thomsen'schen Versuchsdaten im Allgemeinen angefochten sind. Ich habe zuerst nur darauf hingewiesen, dass die mit dem Universalbrenner ausgeführten Bestimmungen wahrscheinlich zu hoch seien, und habe die Richtigkeit dieser, anfangs nur vermuthungsweise ausgesprochenen, Ansicht dann durch den directen Versuch bewiesen. Da die Bestimmung des Wärmewerthes der Oxalsäure aber nieht mit dem Universalbrenner gemacht ist, so beweiset die Uebereinstimmung der von Hrn. Jahn, wie die der jetzt von uns, gefundenen Zahl mit der von Thomsen ermittelten absolut gar nichts für die Richtigkeit der mit dem Universalbrenner gewonnenen Werthe. Wenn die Polemik mit Hrn. 'Thomsen einen, von mir im höchsten Grade beklagten, Grad von Heftigkeit angenommen hat, so wird ein Jeder, der die betreffenden Arbeiten gelesen hat, wissen, von welcher Seite dieser Ton angeschlagen worden ist. 
206 Stohmann, Kleber u. Langbein: Ueber den

\section{$\mathrm{COOH}$ \\ 2. Malonsäure $\dot{\mathrm{C}} \mathrm{H}_{2}$ oder $\mathrm{C}_{3} \mathrm{H}_{4} \mathrm{O}_{4} . \quad 104$. $\dot{\mathrm{COOH}}$}

Käufliche Säure wurde durch freiwillige Verdunstung ihrer ätherischen Lösung umkrystallirt. Farblose Prismen vom Schmelzp. 132\%.

Die Verbrennung der Malonsäure liess sich, ebenso wie die der meisten der folgenden Säuren durch Zugeben von geringer Mengen von Naphtalin leicht einleiten.

Wärmewerth der Malonsäure:

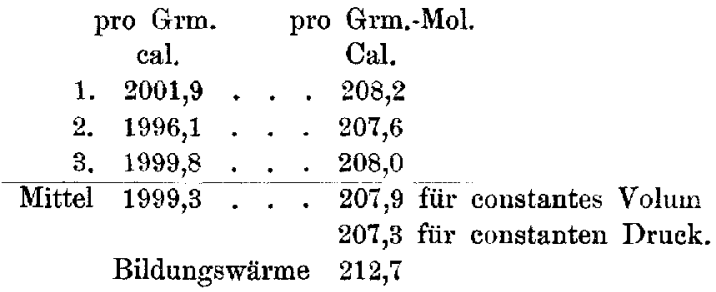

Unsere früheren, mit Kaliumchlorat ausgeführten Bestimmungen hatten 203,8 Cal. ergeben (Abh. I).

Louguinine $\mathrm{e}^{\text {) }}$ bat die Verbrennungswärme der Malonsäure ,zuerst aus der ihres Aethyläthers abgeleitet und dafür 207,6 Cal. gefunden. Durch Verbrennung in der Bombe fand derselbe $^{2}$ ) 208,7 Cal. Obgleich keine specielle Angabe darüber gemacht ist, so lässt sich doch aus der Beschreibung schliessen, dass diese Zahl sich auf constantes Volum beziehe. Auf constanten Druck reducirt, geht der Werth in 208,1 Cal. über.

3. Bernsteinsäure $\stackrel{\stackrel{\mathrm{COOH}}{\dot{\mathrm{C}} \mathrm{H}_{2}}}{\stackrel{\dot{\mathrm{C}} \mathrm{H}_{2}}{\dot{\mathrm{COOH}}}}$ oder $\mathrm{C}_{4} \mathrm{H}_{6} \mathrm{O}_{4} \cdot 118$.

Farblose Krystalle von reiner, käuflicher Bernsteinsäure wurden durch freiwillige Verdunstung ihrer ätherischen Lösung umkrystallisirt und bei $100^{\circ}$ getrocknet. Schmelzp. $180^{\circ}$.

1) Ann. Chim, 8, 142.

2) Compt. rend. 107, 600 . 
Wärmewerth der Säuren der 0xalsäure-Reihe etc. 207

Wärmewerth der Bernsteinsäure:

$$
\begin{aligned}
& \text { pro Grm. pro Grm.-Mol. } \\
& \text { cal. Cal. } \\
& \text { 1. } 3023,2 \text {. . . } 3 \overline{5} 6,7 \\
& \text { 2. } 3027,4 \text {. . } 357,2 \\
& \text { 3. } 3029,7 \text {. . } 357,5 \\
& \text { Mittel } 3026,3 \text {. } \\
& \text { Bildungswärme } \quad 226,2 \\
& 356,8 \text { für constanten Druck. }
\end{aligned}
$$

Unsere früheren Bestimmungen hatten 3506,2 Cal. ergeben (Abh. I).

Louguinine fand in seiner ersten Arbeit, abgeleitet aus dem Diäthyläther, 354,7 Cal., später, beim Verbrennen in der Bombe, 355,8 Cal. für constanten Druck.

Die Bernsteinsäure scheint schon bei wenig erhöhter Temperatur eine partielle Anhydridbildung zu erleiden. Dieselbe Säure, welche zu den vorigen Bestimmungen gedient hatte, ergab, nachdem sie anhaltend zwischen $125^{\circ}$ bis $130^{\circ}$ erwärmt war 359,3 Cal. Nach vorsichtigem Schmelzen stieg ihr Wärmewerth sogar auf 363,1 Cal.

4. Methylmalonsäure oder Isobernsteinsäure

$$
\begin{aligned}
& \mathrm{COOH} \\
& \mathrm{C}<\frac{\mathrm{H}}{\mathrm{CH}_{3}} \text { oder } \mathrm{C}_{4} \mathrm{H}_{6} \mathrm{O}_{4} . \quad 118 \text {. } \\
& \mathrm{COOH}
\end{aligned}
$$

Dargestellt nach dem Verfahren von Conrad ${ }^{1}$ und $Z \ddot{\text { üblin }}{ }^{2}$ ) durch Einwirkung von Jodmethyl auf Natriummalonsäureäther und Zersetzung des entstandenen Produktes mit sehr concentrirter Natronlauge. Die durch Salzsäure abgeschiedene Säure wurde mit Aether ihrer wässrigen Lösung entzogen und, nach dem Verdunsten des Aethers, aus Chloroform, worin sie ziemlich schwer löslich ist, umkrystallisirt. Sie bildet, aus Chloroform krystallisirt, concentrisch vereinte Nadeln, welche bei $130^{\circ}$ schmelzen.

Auf ganz gleiche Weise wurden nach dem allgemeinen Verfahren von Conrad ${ }^{3}$ ) die unten zu besprechenden sonstigen Derivate der Malonsäure dargestellt.
1) Ber. 12, 749 .
) Das. S. 1112.
3) Ann. Chem. 204, 127. 
208 Stohmann, Kleber u. Langbein: Ueber den

Wirmewerth der Isobernsteinsäure:

pro Grm. pro Grm.-Mol.

cal. Cal.

1. 3092,2 . . . 364,9

2. 3100,5 . . 365,9

3. 3097,2 - . 365,5

4. 3101,2 . . 365,9

Mittel 3097,8 . . 365,1 fürr constantes Volum 364,8 für constanten Druck.

Bildungswärme 218,2

$$
\mathrm{CH}_{2} \cdot \mathrm{COOH}
$$

5. Glutarsäure $\dot{\mathrm{CH}}_{2} \quad$ oder $\mathrm{C}_{5} \mathrm{H}_{8} \mathrm{O}_{4}$. 132 .

$$
\mathrm{CH}_{2} \cdot \mathrm{COOH}
$$

Die Glutarsäure wurde erhalten durch Vermischen von Natriummalonsäureäther mit äquivalenten Mengen von $\beta$-Jodpropionsäureäther und Spaltung des entstandenen Produktes mit concentrirter Salzsäure. Die abgeschiedene Säure wurde der Destillation unterworfen und aus Wasser umkrystallisirt. Grosse, bei $97,5^{\circ}$ schmelzende Prismen.

Wärmewerth der Glutarsäure: pro Grm. pro Grm.-Mol. cal. Cal.

1. 3918,7 . . . 517,3

2. 3916,6 . . 517,0

3. 3917,9 . . 517,2

Mittel 3917,7 . . . 517,2 für const. Volum u. Druck. Bildungswärme 298,8

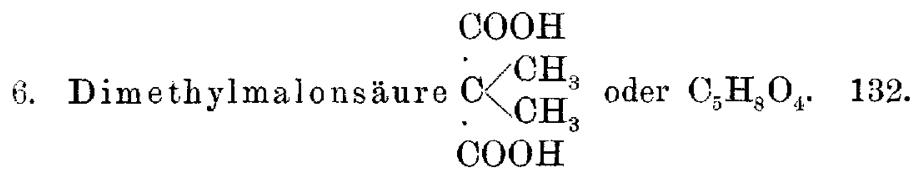

Darstellung nach Conrad's Verfahren (s. Methylmalon. säure). Farblose, bei $186^{\circ}$ schmelzende Krystalle.

Wärmewerth der Dimethylmalonsäure:

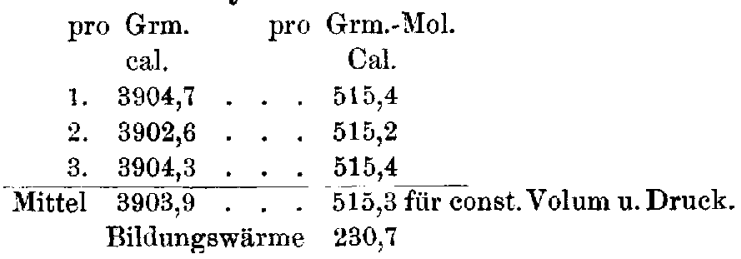


Wärmewerth der Säuren der Oxalsäure-Reihe etc. 209

\section{$\mathrm{COOH}$}

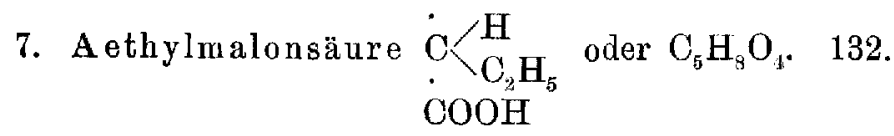

A uf analoge Weise dargestellt wie die Isobernsteinsäure, durch Einwirkung von Jodäthyl auf Natriummalonsäureäther. Aus Chloroform krystallisirt, bildet die Säure grosse farblose Nadeln vom Schmelzp. $112^{\circ}$.

Wärmewerth der Aethylmalonsäure: pro Grm. pro Grm.-Mol.

cal. Cal.

1. 3923,4 . . . 517,9

2. 3914,2 . $\quad 516,7$

3. 3916,8 . . 517,0

4. 3936,6 . . 519,6

5. 3927,9 . . 518,5

Mittel 3928,8 . . . $517,9 \mathrm{f}$, eonst. Volum u.const. Druck. Bildungswärme 228,1

8. Methylbernsteinsäure oder Brenzweinsäure

$\mathrm{COOH}$

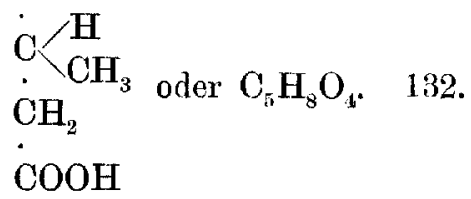

Von Kahlbaum bezogenes Präparat, durch Umkrystallisiren aus Aether gereinigt. Schmelzp. 112".

Wärmewerth der Brenzweinsäure: pro Grm. pro Grm.-Mol. cal. Cal.

1. 3900,9 . . 514,9

2. 3917,1 . . 517,1

3. 3905,8 . . 515,6

4. 3893,1 . . 513,9

5. 3899,5 . . 514,7

6. 3901,2 . . 515,0

Mittel 3902,9 . . 515,2 f. const. Volum u. const. Druek. Bildungswärme 230,8

Louguinine ${ }^{1}$ ) hat die Verbrennungswärme der Brenzweinsäure in der Bombe zu 511,7 Cal. bestimmt.

1) Compt. rend. 107, 600.

Jourual f. prait. Chemie [2] Bd, 40. 
210 Stohmann, Kleber u. Langbein: Ueber den

9. Normale Adipinsäure $\begin{aligned} & \mathrm{CH}_{2} \cdot \mathrm{CH}_{2} \cdot \mathrm{OOOH} \\ & \mathrm{CH}_{2} \cdot \mathrm{CH}_{2} \cdot \mathrm{COOH}\end{aligned}$

Nach dem von Wislicenus ${ }^{1}$ ) angegebenen Verfahren dargestellt. $\beta$-Jodpropionsäure wurde mit fein vertheiltem Silber vorsichtig erhitzt, die Schmelze mit Wasser ausgekocht und die Flüssigkeit mit wenig Salpetersäure verdampft. Das Eindampfen mit sehr verdünnter Salpetersäure wurde mehrere Mal wiederholt, um Jodsubstitutionsprodukte zu zerstören. Die mehrfach aus Wasser umkrystallisirte Säure bildete grosse Prismen, welche bei $150,6^{\circ}$ schmelzen.

Wärmewerth der Adipinsäure:

pro Grm. pro Grm.-Mol.

cal. Cal.

1. 4583,7 . . 669,2

2. 4579,5 . . 668,6

3. 4571,7 . . 667,5

4. 4583,3 . . 669,2

Nititel 4579,8 . 668,6 fïrr constantes Volum 668,9 für constanten Druck.

Bildungswärme 240,1

\section{$\mathrm{cOOH}$}

10. Methyl-Aethylmalonsïure $\begin{aligned} \dot{\mathrm{C}}<\mathrm{C}_{2} \mathrm{H}_{5} & \mathrm{CderC}_{6} \mathrm{H}_{10} \mathrm{O}_{4} .146 . \\ \dot{\mathrm{COOH}} & \text { aO }\end{aligned}$

Darstellung nach Conrad's Verfahren (s. Methylmalonsäure). Die Säure bildet, nach dem Verdampfen ihrer wässrigen Lösung, farblose, bei $119^{\circ}$ schmelzende Krystalle.

Wärmewerth der Methyl-Aothylmalonsïure: pro Grm. pro Grm.-Mol.

cal. $\quad \mathrm{Cal}$.

1. 4604,8 . . 672,3

2. 4605,1 . . 672,3

3. 4600,5 . . 671,7

4. $45^{4} 9,8$. . 671,6

Mittel 4602,6 . $\quad 672,0$ für constantes Volum

Bildungswärme 236,7 672,3 für constanten Druek.

1) Ann. Chem 149, 220. 


\section{$\mathrm{COOH}$}

11. Propylmalonsäure $\begin{aligned} \dot{\mathrm{C}} & \underset{\mathrm{C}}{\mathrm{C}} \mathrm{C}_{3} \mathrm{H}_{7} \\ & \text { oder } \mathrm{C}_{6} \mathrm{H}_{10} \mathrm{O}_{4} \text {. } 146 .\end{aligned}$

Nach Conrad's Verfahren (s. Methylmalonsäure) dargostellt, wurde die Säure aus Wasser krystallisirt. Schmelzp. 84".

Wärmewerth der Propylmalonsïure:

$$
\begin{aligned}
& \text { pro Grm. pro Grm.-Mol. } \\
& \text { cal. Cal. } \\
& \text { 1. } 4617,9 \text {. . } 674,2 \\
& \text { 2. } 4622,0 \text {. . } 674,8 \\
& \text { 3. } 4624,1 \text {. . } 675,1 \\
& \text { Mittel 4621,3 . . . 674,7 für constantes Volum } \\
& 675,0 \text { für constanten Druck. }
\end{aligned}
$$

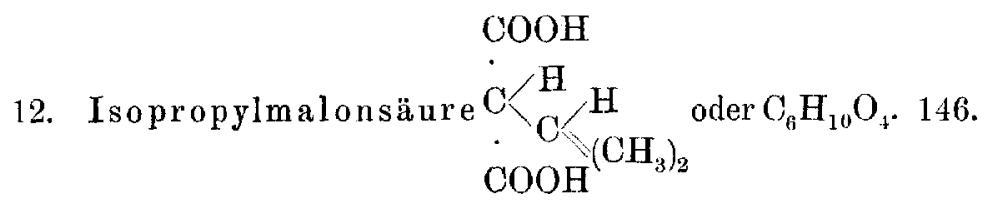

Darstellung wie bei den übrigen substituirten Malonsäuren, nach dem Conrad'schen Verfahren. Farblose, bei $87^{\circ}$ schmelzende Krystalle.

Wärmewerth der Isopropylmalonsäurc: pro Grm. pro Grm. Mol.

$$
\text { eal. Cal. }
$$

1. 4620,9 . . 674,7

2. 4623,3 . . 675,0

3. 4628,1 . . 675,7

4. 4618,2 . . 674,3

Mittel 4622,6 - . fi74,9 für constantes Volum 675,2 für constanten Druck.

Bildungswärme $\quad 233,8$ 
212 Stohmann, Kleber u. Langbein: Ueber den

13. Symmetrische Dimethylbernsteinsäure oder $\mathrm{COOH}$

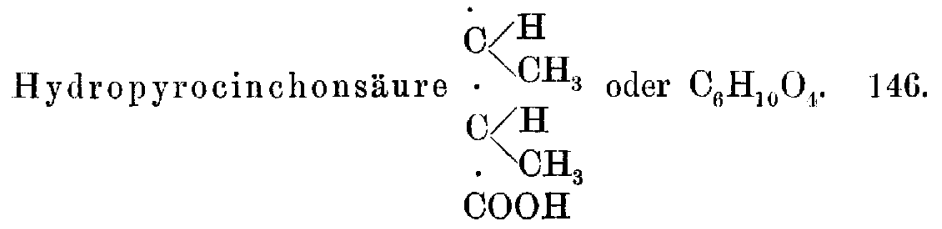

Dargestellt nach Bischoff und $\mathrm{Rach}^{1}$ ) durch Einwirkung von Natriummethylmalonsäureäther auf $\alpha$-Brompropionsäureäther und Spaltung des Produktes mit concentrirter Salzsüure. In Wasser ziemlich schwer lösliche Prismen, schmilzt bei $192^{\circ}$. Liefert beim Destilliren (Siedep. $232^{\circ}$ ) ein bei $83^{\circ}$ schmelzendes Anliydrid. Beim Unkrystallisiren aus einer Mischung von Benzol und Ligroin wurde das Anhydrid in Form schöner weisser Blättchen erhalten.

Wärmewerth der symmetrischen Dimethylbernsteinsäure: pro Grm. pro Grm.-Mol. eal. Cal.

14. Unsymmetrische Dimethylbornsteinsäure $\mathrm{COOH}$

$\dot{\mathrm{CH}}_{2}$ $\dot{\mathrm{C}} \mathrm{CH}_{3}$ oder $\mathrm{C}_{6} \mathrm{H}_{10} \mathrm{O}_{1} \cdot 146$.
$\mathrm{COOH}$

Dargestellt nach dem Verfahren von Leuckart ${ }^{2}$ ) durch Einwirkung von $\alpha$-Bromisobnttersïureitther auf Natriummalonsäureäther und Kochen des entstandenen Isobutenyltricarbonsäureäthers mit concentrirter Salzsäure. Farblose, leicht in Wasser lösliche, bei $140^{\circ}$ schmelzende Prismen.

1) Ann. Chem. 934, 54.

2) Ber. 15, 2350 . 
Wärmewerth der Säuren der Oxalsäure-Reihe etc. 213

Wärmewerth der unsymmetrischen Dimethylbernsteinsäure: pro Grm. pro Grm.-Mol. cal. Cal.

1. 4592,9 . . 670,6

2. 4598,9 . . . 671,4

3. 4605,8 . . 672,4

4. 4597,4 . . 671,2

Mittel 4598,8 . . 671,4 für constantes Volum Bildungswärme 237,3 671,7 für constanten Druck.

$\mathrm{COOH}$

$\mathrm{CH}_{2}$

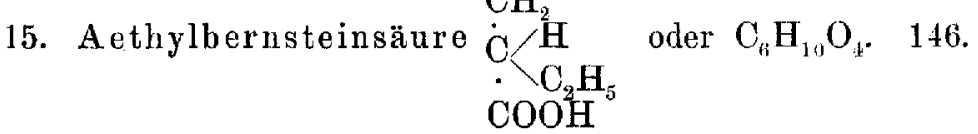

Dargestellt nach dem Verfahren von Huggenberg. ${ }^{1}$ ) Aethylacetbernsteinsäureäther wurde durch alkoholisches Kali zersetzt. Die aus dem Kaliumsalz abgeschiedene Säure wurde in wenig Aether gelöst, wobei etwas mitgebildete Bernsteinsäure zurückblieb, und aus heissem Benzol umkrystallisirt. Farblose, feine Nadeln, bei $98^{\circ}$ schmelzend.

Wärmewerth der Aethylbernsteinsüure: pro Grm. pro Grm.-Mol. cal. Cal.

1. 4604,4 . . 672,2

2. 4601,4 . . 671,8

3. 4600,4 . - 671,7

Mittel 4602,1 - 671,9 für constantes Volum

Bildungswärme 236,8 672,2 für constanten Druck.

COOH

C $\angle \mathrm{H}$

16. $\alpha$-Methylglutarsäure $\mathrm{CH}_{2}$ oder $\mathrm{C}_{0} \mathrm{H}_{10} \mathrm{O}_{3}$. 146 . $\dot{\mathrm{CO}}_{2}$
$\mathrm{COH}$

Bei der Darstellung wurde genau das von $K_{\text {rekeler }}^{2}$ ) angegebene Verfahren innegehalten, und die gewonnene Säure

1) Ann. Chem. 192, 146.

2) Ber. 19, 3269 . 
214 Stohmann, Kleber n. Langbein: Ueber den

mehrfach aus Wasser umkrystallisirt. Sie wurde in gut ausgebildeten, glasglänzenden, bei $75,8^{\circ}$ schmelzenden Prismen erhalten.

Wärmewerth der $\alpha$-Methylglutarsäure:

$$
\begin{aligned}
& \text { pro Grm. pro Grm.-Mol. } \\
& \text { cal. Cal. } \\
& \text { 1. } 4593,5 \text {. . . } 670,7 \\
& \text { 2. } 4590,7 \text {. . . } 670,2 \\
& \text { 3. } 4592,4 \text {. . . } 670,5 \\
& \text { Mittel 4592,2 . . . } 670,5 \text { für constantes Volum } \\
& 670,8 \text { für constanten Druck. }
\end{aligned}
$$

$$
\begin{aligned}
& \text { 17. Normale Pimelinsäure } \mathrm{CH}_{2}\left\{\begin{array}{l}
\mathrm{CH}_{2} \cdot \mathrm{CH}_{2} \cdot \mathrm{COOH} \\
\mathrm{CH}_{2} \cdot \mathrm{CH}_{2} \cdot \mathrm{COOH}
\end{array}\right. \\
& \text { oder } \mathrm{O}_{7} \mathrm{H}_{12} \mathrm{O}_{1} \cdot 160 .
\end{aligned}
$$

Dargestellt nach dom Verfahren von Dale und Schorlemmer ${ }^{1}$ ) durch Oxydation von reinem Suberon. Letzteres wurde erhalten, indem das trockne Calciumsalz der Korksäure in einer weiten, am einen Ende geschlossenen Röhre der trocknen Destillation unterworfen, und das ïbergehende Gemisch von Hexan und Suberon, durch Schütteln mit einer Lösung von primärem Natriumsulfit, in die sich in Blättchen ausscheidende Sulfitverbindung des Suberons verwandelt wurde. Diese wurde mit A ether bis zum Farbloswerden gewaschen, dann mit Natriumcurbonat zersetzt, und das Suberon mit Aother ausgeschüttelt. Nach dem Verdampfen des Aethers wurde das Suberon mit Salpetersäure oxydirt. Nach dem Verdampfen der letzteren krystallisirte die $\alpha$-Pimelinsäure aus. Sie wurde abgepresst, zuerst aus Wasser, dann aus heissem Benzol umkrystallisirt und bildete dünne Tafeln, deren Schmelzpunkt bei $104^{\circ} \mathrm{lag}$. Der Schmelzpunkt liegt daher um $4^{0}$ höher als der von Dale und Schorlemmer angegebone, er nähert sich aber sehr dom Schmelzpunkt $105,5^{\circ}$ bis $106^{\circ}$, welcher von Gantter und $\mathrm{Hell^{2 }}$ ) für die aus Ricinusöl erhaltene, als $\beta$-Pimelinsäure bezeichnete Säure angegebcn wird. Unsere Säure theilte mit letzterer auch die als Kriterion der Reinheit angegebene Eigenschaft: Die geschmolzene und erstarrte Säure zersprang bei

1) Ann. Chem. 199, 144.

2) Ber. 17, 2212 . 
Wärmewerth der Säuren der Oxalsäure-Reihe ete. 215

der leisesten Berührung zu Trümmern unter Umherschleudern der Fragmente. Es ist daher nicht unwahrscheinlich, dass die bisher als $\alpha$ - und $\beta$-Pimelinsäuren unterschiedenen Säuren identisch sind.

Wärmewerth der Pimelinsäure:

pro Grm. pro Grin.-Mol.

eal. Cal.

1. 5181,5 . . . 829,0

2. 5180,7 . . 828,9

Mittel 5181,1 . . 829,0 für constantes Volum

829,6 für constanten Druck.

Bildungswärme 242,4

18. Korksäure oder Suberinsäure

${ }_{1} \mathrm{CH}_{2} \cdot \mathrm{CH}_{2} \cdot \mathrm{CH}_{2} \cdot \mathrm{COOH}$

$\mathrm{CH}_{2} \cdot \mathrm{CH}_{2} \cdot \mathrm{CH}_{2} \cdot \mathrm{COOH}$ orler $\mathrm{C}_{8} \mathrm{H}_{14} \mathrm{O}_{4} \cdot 174$.

Von Schuchardt bezogenes Präparat, wurde durch Umkrystallisiren weiter gereinigt, Schmelzp. $141^{0}$.

Wärmewerth der Korksäure:

pro Grin, pro Grm. Mol.

cal, Cal.

1. 5660,0 . . . 984,8

2. 5656,4 . . 984,2

3. 5658,8 . . . 984,6

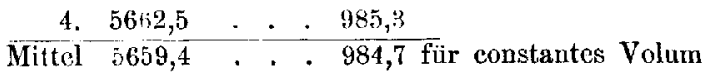

Bildungswärme 249,4

985,6 für constanten Druck

Louguinine ${ }^{1}$ ) fand für die Verbrennungswärme der
ksäure 992,4 Cal. für constantes Volum oder 993,3 Cal. Korksäure 992,4 Cal. für constantes Volum oder 993,3 Cal. für constanten Druck.

19. Azelänsäure oder Lepargylsäure

$\mathrm{CH}_{2}\left\{\begin{array}{l}\mathrm{CH}_{2} \cdot \mathrm{CH}_{2} \cdot \mathrm{CH}_{2} \cdot \mathrm{COOH} \\ \mathrm{CH}_{2} \cdot \mathrm{CH}_{2} \cdot \mathrm{CH}_{2} \cdot \mathrm{COOH}\end{array}\right.$ oder $\mathrm{O}_{9} \mathrm{H}_{1 ;} \mathrm{O}_{4} \cdot 188$.

Von Schuchardt bezogen, aus heissem Wasser umkrystallisirt. Farblose, bei $106^{\circ}$ bis $106,5^{\circ}$ schmelzende Blätter.

1) Compt. rend. 107, 600 . 
216 Stohmann, Kleber u. Langbein: Ueber den

Wärmewerth der Azclaïnsäure:

pro Grm. pro Grm.-Mol.

cal. Cal.

1. 6061,7 . . 1139,6

2. 6070,3 . . 1141,2

3. 6067,0 . . . 1140,6

4. 6059,4 . . 1139,2

Mittel 6064,6 . . 1140,1 für constantes Volum 1141,3 für constanten Druck.

Bildungswärme 256,7

20. Sebacinsäure $\left\{\begin{array}{l}\mathrm{CH}_{2} \cdot \mathrm{CH}_{2} \cdot \mathrm{CH}_{3} \cdot \mathrm{CH}_{2} \cdot \mathrm{COOH} \\ \mathrm{CH}_{2} \cdot \mathrm{CH}_{2} \cdot \mathrm{CH}_{2} \cdot \mathrm{CH}_{2} \cdot \mathrm{COOH}\end{array}\right.$ oder $\mathrm{C}_{10} \mathrm{H}_{18} \mathrm{O}_{4} .202$.

Dargestellt nach dem Verfahren von Witt') durch Erhitzen von Ricinusölseife. Die Säure wurde aus ziemlich concentrirter Salpetersäure, durch welche sie nicht zersetzt wird, umkrystallisirt. Sie bildet dünne, farblose, bei $133^{\circ}$ schmelzonde Blättchen.

Wärmewerth der Sebacinsäure:

pro Grm. pro Grm.-Mol.

cal. Cal.

1. 6409,0 . . . 1294,6

2. 6416,5 . . . 1296,1

3. $6411,6 \div$. . 1295,1

Mittel 6412,4 . . . 1295,3 für constantes Volum

Bildungswärme 264,2

1296,8 für constanten Druck.

Louguinine fand 1295,6 Cal. für constantes Volum oder 1297,1 Cal. für constanten Druck.

21. Eumarsäure

HOOC. CH

$$
\text { HC. COOH oder } \mathrm{C}_{4} \mathrm{H}_{4} \mathrm{O}_{4} .116 .
$$

Von Kahlbaum bezogenes, sehr reines Präparat von uns aus Wasser umkrystallisirt.

Wärmewerth der Fumarsäure:

pro Grm. pro Grin.-Mol.

$$
\text { cal. Cal. }
$$

1. 2757,3 . . 319,8

2. 2771,0 . . 321,4

3. 2767,5 . . . 321,0

4. $2764,7: \quad 320,7$

Mittel $2765,1.320,7$ für constantes Volum 320,1 für constanten Druek.

Bildungswärme $\quad 193,9$

1) Ber. 7, 220. 
Wärmewerth der Säuren der Oxalsäure-Reihe etc. 217

Louguinine ${ }^{1}$ ) fand für Fumarsäure 318 ,2 Cal. für constantes Volum, woraus sich 317,6 Cal. für constanten Druck ergiebt.

\section{Maleïnsäure $\underset{\mathrm{HC}}{\dot{\mathrm{C}} . \mathrm{COOH}}$ oder $\mathrm{C}_{4} \mathrm{H}_{4} \mathrm{O}_{2}, 116$.}

Von Kahlbaum bezogen, aus ätherischer Lösung durch Darüberleiten von getrockneter Luft umkrystallisirt. Schmelzpunkt $130^{\circ}$.

Wärmewerth der Maleïnsäure:

$$
\text { pro Grm. pro Grm.-Mol. }
$$

cal. Cal.

$$
\begin{aligned}
& \text { 1. } 2817,5 \text {. . } 326,8 \\
& \text { 2. } 2819,7 \text {. . . } 327,1 \\
& \text { 3. } 2817,9 \text {. . . } 326,9 \\
& \text { Mittel 2818,4 . . . } 326,9 \text { für constantes Volum } \\
& \text { 326,3 für constanten Druck. } \\
& \text { Bildungswärme } 187,7
\end{aligned}
$$

Nach Louguinine's Bestimmung ist die Verbrennungswärme der Maleïnsäure bei constantem Volum 331,7 Cal. oder bei constantem Druck 331,1 Cal.

Uebersicht der in Vorstehendem ermittelten Werthe:

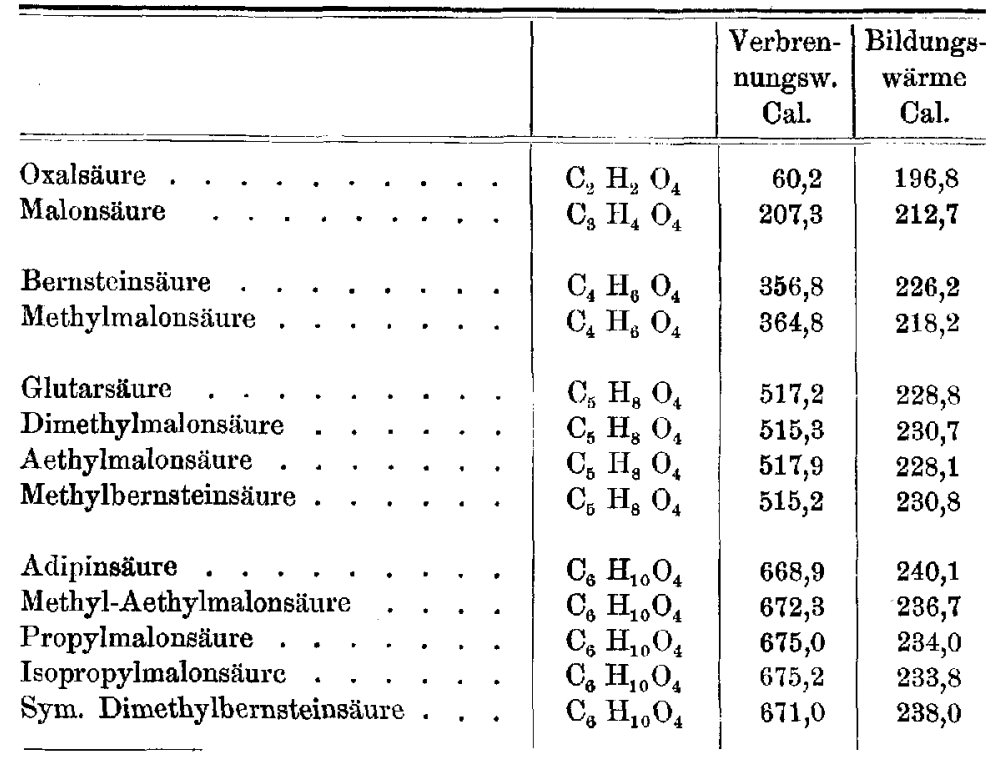

1) Compt. rend. 106, 1289. 


\begin{tabular}{|c|c|c|c|c|c|c|c|c|c|c|}
\hline & & & & & & & & & $\begin{array}{c}\text { Verbren- } \\
\text { nungsw } \\
\text { Cal. }\end{array}$ & $\begin{array}{c}\text { Bildungs- } \\
\text { wärme } \\
\text { Cal. }\end{array}$ \\
\hline \multicolumn{8}{|c|}{ Unsym. Dimethylbernsteiusiure . } & $\mathrm{C}_{6} \mathrm{H}_{10} \mathrm{O}_{4}$ & 671,7 & 237,3 \\
\hline Aethylbernstei & usäu & & & & . & & . & $\mathrm{C}_{6} \mathrm{H}_{10} \mathrm{O}_{4}$ & 672,2 & 236,8 \\
\hline Methylglutars: & iure & . & & & 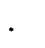 & & . & $\mathrm{O}_{6} \mathrm{H}_{10} \mathrm{O}_{4}$ & 670,8 & 238,2 \\
\hline Pimelinsäure & . & . & & - & . & & . & $\mathrm{C}_{7} \mathrm{H}_{12} \mathrm{O}_{4}$ & 829,6 & 242,4 \\
\hline Korksäure . & & . & & & ${ }^{\circ}$ & & • & $\mathrm{C}_{8} \mathrm{II}_{14} \mathrm{O}_{4}$ & 985,6 & 249,4 \\
\hline Azolaïnsäure & . & . & . & • & . & & . & $\mathrm{O}_{9} \mathrm{H}_{16} \mathrm{O}_{4}$ & 1141,3 & 256,7 \\
\hline Subacimsäure & . & . & - & 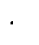 & $\cdot$ & & . & $\mathrm{C}_{10} \mathrm{HI}_{18} \mathrm{O}_{4}$ & 1296,8 & 264,2 \\
\hline Fumarsäule & . & . & • & • & - & & & $\mathrm{C}_{4} \mathrm{H}_{4} \mathrm{O}_{4}$ & 320,1 & 193,9 \\
\hline Maleïusäure & , : & , & . & . & . & & . & $\mathrm{C}_{4} \mathrm{H}_{4} \mathrm{O}_{4}$ & 326,3 & 187,7 \\
\hline
\end{tabular}

1. Is omerie.

Es ist bisher bei thermochemischen Untersuchungen noch keine so grosse Zahl von vergleichbaren Beobachtungen gebracht, wie dic vorstehende Uebersicht sie bietet. Haben wir bisher meistens die structurisomeren Verbindungen in ihrem Wärmewerthe als nahezu gleich gefunden, so ist dieses hier nicht in gleichem Masse der Fall, und es lassen sich deutlich zwei Gruppen unterscheiden, deren Glieder entweder der Malonsäure - oder der Bernsteinsäure-Reihe angehören, und von denen die der Malonsäure-Reihe einen böheren Wärmewerth besitzen, als die ihnen isomeren der BernsteinsäureReihe.

Bei den Säuren $\mathrm{C}_{4} \mathrm{H}_{6} \mathrm{O}_{4}$ haben wir:

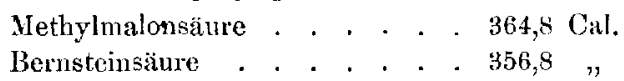

Bei den Siiuren $\mathrm{O}_{5} \mathrm{H}_{8} \mathrm{O}_{4}$ tritt der Unterschied weniger scharf hervor, insofern als der Wärmewerth der Dimethylmalonsäure und der Methylbernsteinsäure gleich ist; andererseits macht sich die Verschiedenheit bei den folgenden beiden Säuren in gleichem Sinne wieder geltend:

$$
\begin{aligned}
& \text { Aethylmalonsäure . . . . . . 517,9 Cal. } \\
& \text { Methylbernsteinsäure . . . . . 515,2 " }
\end{aligned}
$$

In der dritten Säurereihe $\mathrm{C}_{6} \mathrm{H}_{10} \mathrm{O}_{4}$ sind wieder zwei der zur Malonsäure- und zur Bernsteinsäure-Reihe gehörende Glieder 
Wärmewerth der Säuren der Oxalsäure-Reihe etc. 219

sich gleich, die Methyl-Aethylnalonsäure und die Aethylbernsteinsäure, dagegen entsprechen aber jedesmal zwei Säuren dem Verhalten der Malonsäure und Bernsteinsäure, nämlich:

$$
\begin{aligned}
& \text { Propylmalonsäure . . . . . , 675,0 Cal. } \\
& \text { Isopropylmalonsäure . . . . . 675,2 " } \\
& \text { Sym, Dimethylbernsteinsäure . 671,0 Cal. } \\
& \text { Unsym. Ditncthylbernsteinsäure . 671,7, , }
\end{aligned}
$$

Dieselben Differenzen finden wir, wie gleich gezeigt werden wird, in der Homologie wieder.

Unter den hier in Betracht kommenden Verbindungen haben wir zwei geometrisch isomere Säuren, die aber thermisch ein selur verschiedenes Verhalten zeigen.

$$
\begin{aligned}
& \text { Maleinsäure . . . . . . 326,3 Cal. } \\
& \text { Fumarsăure . . . . . . . . 320,1 " }
\end{aligned}
$$

Ganz in glcichem Sime, nur noch erheblich grösser, gestalten sich die Differenzen bei diesen beiden Süuren, wem man die vou Louguinine gefundenen Werthe der Ver-

\begin{tabular}{|c|c|c|c|}
\hline & & & Differenz: \\
\hline Oxalsiaure & 60,0 & $\mathrm{Ca}$ & 148,7 \\
\hline Malonsäure . & 208,7 & $"$ & 147,4 \\
\hline Bernsteinsäure & 356,1 & & 155,6 \\
\hline Brenzweinsïure & 511,7 & & $160,2 \times 3$ \\
\hline Korksäure . . & 992,4 & & $151,6 \times 2$ \\
\hline Sebacinsäure & 1295,6 & & \\
\hline
\end{tabular}
gleichung zu Grunde legt.

\section{Homologie.}

Louguinine ${ }^{1}$, welcher einige der hierher gehörenden Säuren untersucht hat, berechnet folgende Zahlen für die Homologen:

Louguinine knïpft daran folgende Bemerkungen: „Nach „dieser Uebersicht würde es erscheinen, dass Oxal-, Malon„und Bernsteinsïure eine homologe Reihe bilden, während „Kork- und Sebacinsäure einer anderen homologen Reihe an"gehören; die Brenzweinsäure nimmt eine anormale Stellung „in beiden Reihen ein. Da die Verbrennungswärme mit grosser „Genauigkeit ermittelt war, so muss diese Anomalie, nach

1) Compt. rend. 107, 600 . 
220 Stohmann, Klebor u. Langbein: Ueber den

„mciner Meinung, der Structur des Moleküles dieser Säure zu"geschrieben werden, durch welche diese Säure von ihren "höheren und niederen Homologen verschieden gemacht wird."

Die ganzen Unregelmässigkeiten, welche sich in den Zahlen von Louguinine zeigen und welche, wenn auch nicht in so ausgeprägtem Sinne, sich in den von uns ermittelten Werthen wiederholen, verschwinden sofort, wenn man in dieser Süuregruppe zwei verschiedene Anfangsglieder, worauf schon oben hingewiesen ist, die Malonsäure und die Bernsteinsäure, annimmt und zunächst deren Derivate mit einander vergleicht. Es finden sich dann nirgends die extremen Werthe, sondern es bewegen sich alle Zahlen in den auch sonst überall beobachteten Grenzen, auch dic Brenzweinsäure erweiset sich in voller Uebereinstimmung mit ihren wirklichen Homologen.

Malonsäure-Derivate:

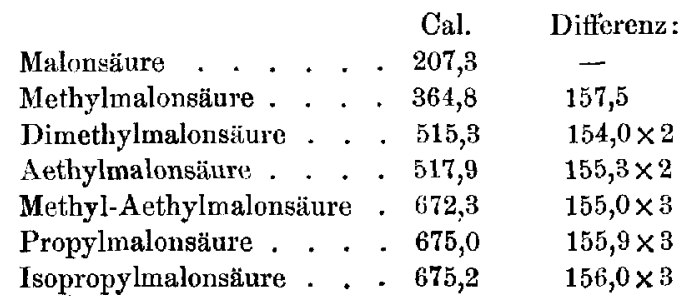

Bernsteinsäure-Derivate:

\begin{tabular}{|c|c|c|}
\hline Bernsteinsäure & $\begin{array}{c}\text { Cal. } \\
356,8\end{array}$ & Differenz: \\
\hline Methylbernsteinsäure & 515,2 & 158,4 \\
\hline Symm. Dimethylbernsteins. & 671,0 & $157,1 \times 2$ \\
\hline Unsymm. Dimethylbernstein & 671,7 & $157,5 \times 2$ \\
\hline Aethylbernsteinsäure . . & 672,2 & $157,7 \times 2$ \\
\hline
\end{tabular}

Bei den höheren Homologen der normalen Säuren zeigen sich die regelmässigsten Verhältnisse, wenn man einerseits die Säuren mit ungeraden Zahlen der Kohlenstoffatome und andererseits die Säuren mit geraden Zahlen der Kohlenstoffatome unter einander vergleicht:

\begin{tabular}{|c|c|c|c|}
\hline & & Cal. & \\
\hline Malonsäure & $\mathrm{C}_{3} \mathrm{H}_{4} \mathrm{O}_{4}$ & 207,37 & $155,0 \times 2$ \\
\hline Glutarsäure & $\mathrm{C}_{5} \mathrm{H}_{3} \mathrm{O}_{4}$ & 517,2 & $1562 \times 2$ \\
\hline Pimelinsäure & $\mathrm{O}_{7} \mathrm{H}_{12} \mathrm{O}_{4}$ & 829,6 & $1554 \times 2$ \\
\hline Azaleïnsäure & $\mathrm{C}_{9} \mathrm{H}_{16} \mathrm{O}_{4}$ & $1141,3^{\rfloor}$ & \\
\hline
\end{tabular}


Cal.

$\begin{array}{lllr}\text { Bernsteinsäure } & \mathrm{C}_{4} \mathrm{H}_{6} \mathrm{O}_{4} & 356,8 \\ \text { Adipinsäure } & \mathrm{C}_{6} \mathrm{H}_{10} \mathrm{O}_{4} & 668,9 & 156,1 \times 2 \\ \text { Korksäure } & \mathrm{C}_{8} \mathrm{H}_{14} \mathrm{O}_{4} & 985,6 \\ \text { Sebacinsäure } & \mathrm{C}_{10} \mathrm{H}_{16} \mathrm{O}_{4} & 1296,8^{-} & 155,6 \times 2 \\ \text { S. } & & \end{array}$

Die Annahme von zwei verschiedenen Reihen von Säuren rechtfertigt sich auch durch eine weitere Eigenschaft derselben. Es ist schon von Baeyer ${ }^{1}$ ) und von $\mathrm{Hen} \mathrm{ry}^{2}$ ) auf die Verschiedenheiten der Schmelzpunkte der hierher gehörenden Säuren hingewiesen und beide Forscher haben gezeigt, dass die einzelnen Glieder der ganzen Säurercihe unter einander in dieser Beziehung scheinbar gar keine Regelmässigkeiten darbieten, während ganz bestimmte Schmelzpunktsintervalle sich bemerklich machen, wenn man einerseits die Säuren mit ungeraden und andererseits die Säuren mit geraden Kohlenstoffatomzahlen mit einander vergleicht. Dieselben Regelmässigkeiten finden sich in den Verbrennungswärmen wieder.

Die Oxalsäure füllt in ihrem Wärmewerth gänzlich aus der Reihe der Homologen heraus. Vergleichen wir ihre Verbrennungswärme, einerseits mit der der Malonsïure und dann mit der der Bernsteinsäure:

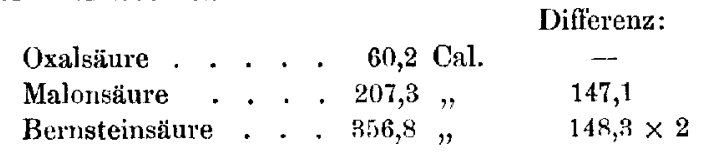

so sind in beiden Fällen die Differenzen gegen die übrigen Glieder der Reihen so gross, dass hier besondere Umstände walten müssen.

Aehnliche Verhältnisse scheinen bei den einbasischen Fettsäuren vorzukommen. Wir haben früher (Abh. IV ${ }^{3}$ ) den Wärmewerth der Ameisensäure, welcher nach der damals von uns angewandten Methode nicht direct bestimmbar war, auf Grund der Homologie-Differenz zu 59,0 Cal. angenommen und hatten für diesen Werth eine Bostätigung in der von Thomsen ermittelten Zahl (60,2 Cal.) gefunden. Nach neueren von $\mathrm{Jahn}^{4}$ ) ausgeführten Messungen, wobei sich für Ameisensäure

1) Ber. 10, 1286 .

${ }^{2}$ ) Compt. rend. 100, 60; Ber. 18, Ref. 59.

3) Dies. Joum. [2] 32, 419.

4) Ann. Phys. [2] 37, 408. 
222 Stohmann, Kleber u. Langbein: Ueber den

62,87 Cal. ergab, scheint aber sowohl unsere wie Thomsens Zahl um etwas zu gering zu sein. Vergleicht man Jahn's Zahten:

$$
\begin{array}{llrc} 
& & & \text { Differenz: } \\
\text { Ameisensäure . . . } & 62,87 \text { Cal. } & - \\
\text { Essigsä̆nre . . . . } & 208,81, & 145,9
\end{array}
$$

so zejgt sich auch hier die Abweichung van dem HomologieWerthe und zwar ganz in gleicher Richtung wie wir es oben für die zweibasischen Säuren gefunden haben.

Es ergiebt sich daher hieraus, dass die Anfangsglieder der homologen Fettsäuren, und zwar sowohl die einbasischen wie die zweibasischen, gegenüber den höhoren Gliedern eine besondere Stellung einnehmen. Es steht dieses in vollstem Einklange mit ihrem sonstigen physikalischen Verhalten. (Vergl. Ostwald, Affinitätsgrössen organischer Säuren. ${ }^{1}$ )

Ganz ähnliche Verhältnisse sind für die Anfangsglieder der homologen Reihen bereits mehrfach beobachtet, so z. B. in neuester Zeit von Berthelot und Petit ${ }^{2}$ ) für die Nitrile.

3. Verhalten der einbasischen zu den zweibasischen Säuren.

In Abhandlung XVIII haben wir gezeigt, dass der Wärmewerth der einbasischen Benzoësäure, der zweibasischen Phtal. sïure und der dreibasischen Trimesinsïure wesentlich gleich sei, dass also der Wärmewerth sich nicht merklich ändere, wenn in einer einbasischen Säure ein Wasserstoffatom durch eine Carboxylgruppe ersetzt wird.

Ganz ähnliche Verhältnisse ergeben sich beim Vergleiche der Säuren der Ameisen- und Essigsäurereihe mit denen der Oxalsäurereihe.

Un die Vergleichung ausführen zu können, sind beide Säuren auf gleichen Aggregatzustand zu bringen. Aus den Bestimmungen von Pettersson ${ }^{3}$ ) ergiebt sich die Schmelzwärme der Ameisensäure und der Essigsäure zu -2,6 Cal. für das Molekül, unsere im Eiscalorimeter ausgeführten Bestim-

\footnotetext{
1) Zeitschr. physik. Chem. 3, 174, 281.

2) Compt. rend. 108, 1217.

3) Dies. Journ. [2] 32, 298.
} 
mungen (dbh. $\mathrm{II}^{1}$ ) hatten für Laurinsäure 8,7 Cal. und für Myristinsäure 10,7 Cal. ergeben. Combinirt man diese Zahlen miteinander, so ergiebt sich als mittlere Schmelzwärme - 0,048 Cal. für das Gramm jeder der Säuren. Von dieser Zahl haben wir hier, wie auch früher, Gebrauch gemacht, um den für den flüssigen Zustand bekannten Wärmewerth der Sïuren auf den festen Aggregatzustand zu reduciren.

Zum Vergleich nehmen wir für Ameisensäure und Essigsäure die von Jahn ermittelten Zahlen, für die übrigen Säuren unsere eignen Zahlen (Abh. IV $\left.{ }^{2}\right)$ ), deren Richtigkeit durch die von Louguinine ausgeführten Bestimmungen erwiesen ist (Abh. XIV). ${ }^{3}$ )

Hiernach ergeben sich folgende Beziehungen:

\begin{tabular}{|c|c|c|c|}
\hline \multirow{3}{*}{$\begin{array}{l}\text { Amcisensäure, fest } \\
\text { Oxalsäure }\end{array}$} & \multirow{3}{*}{$\begin{array}{l}\mathrm{CH}_{2} \mathrm{O}_{3} \\
\mathrm{C}_{2} \mathrm{H}_{2} \mathrm{O}_{4}\end{array}$} & \multirow{2}{*}{$\begin{array}{l}\text { Cal. } \\
60,3\end{array}$} & \multirow{2}{*}{$\begin{array}{c}\text { Verhältnisszahl } \\
\qquad 100\end{array}$} \\
\hline & & & \\
\hline & & 60,2 & 99,8 \\
\hline Essigsäure, fest & $\mathrm{C}_{22} \mathrm{H}_{4} \mathrm{O}_{2}$ & 206,2 & 100 \\
\hline Malonsäure & $\mathrm{C}_{3} \mathrm{H}_{4} \mathrm{O}_{4}$ & 207,3 & 100,5 \\
\hline Propionsäure, fest & $\mathrm{C}_{3} \mathrm{H}_{6} \mathrm{O}_{2}$ & 364,3 & 100 \\
\hline Bernsteinsäure & $\mathrm{C}_{4} \mathrm{H}_{6} \mathrm{O}_{4}$ & 356,8 & 97,9 \\
\hline Propionsïure, fest & $\mathrm{C}_{3} \mathrm{H}_{6} \mathrm{O}_{2}$ & 364,3 & 100 \\
\hline Isobernsteinsäure & $\mathrm{C}_{4} \mathrm{H}_{6} \mathrm{O}_{4}$ & 364,8 & 100,1 \\
\hline Buttersäure, fest & $\mathrm{C}_{4} \mathrm{H}_{8} \mathrm{O}_{2}$ & 518,5 & 100 \\
\hline Glutarsäure & $\mathrm{C}_{5} \mathrm{H}_{9} \mathrm{O}_{4}$ & 517,2 & 99,7 \\
\hline Buttersüure, fest & $\mathrm{C}_{4} \mathrm{H}_{8} \mathrm{O}_{2}$ & $51.8,5$ & 100 \\
\hline Dimethylmalonsäure & $\mathrm{C}_{5} \mathrm{H}_{3} \mathrm{O}_{4}$ & 515,3 & 99,4 \\
\hline Buttersäure, fest & $\mathrm{O}_{4} \mathrm{H}_{3} \mathrm{O}_{2}$ & 518,5 & 100 \\
\hline Aethylmalonsäure & $\mathrm{C}_{5} \mathrm{II}_{8} \mathrm{O}_{4}$ & 517,9 & 99,8 \\
\hline Buttersäure, fest & $\mathrm{O}_{4} \mathrm{H}_{8} \mathrm{O}_{4}$ & 518,5 & 100 \\
\hline Methylbernsteinsiuure & $\mathrm{C}_{5} \mathrm{H}_{8} \mathrm{O}_{4}$ & 515,2 & 99,4 \\
\hline Valeriansäure, fest & $\mathrm{C}_{5} \mathrm{H}_{10} \mathrm{O}_{2}$ & 671,8 & 100 \\
\hline Adipinsäure & $\mathrm{C}_{6} \mathrm{H}_{10} \mathrm{O}_{4}$ & 668,9 & 99,6 \\
\hline Valeriansäure, fest & $\mathrm{C}_{5} \mathrm{H}_{10} \mathrm{O}_{2}$ & 671,8 & 100 \\
\hline Methyl-Aethylmalons. & $\mathrm{C}_{3} \mathrm{H}_{10} \mathrm{O}_{4}$ & 672,3 & 100,1 \\
\hline
\end{tabular}

1) Dies. Journ. [2] 32, 80.

2) Das. 32, 418.

3) Das. 36, 139. 
224 Stohmann, Kleber u. Langbein: Ueber den

$\begin{array}{lccc} & & \text { Cal. } & \text { Verhältnisszahl: } \\ \text { Valeriansäure, fest } & \mathrm{C}_{6} \mathrm{H}_{10} \mathrm{O}_{2} & 671,8 & 100 \\ \text { Propylmalonsäure } & \mathrm{C}_{6} \mathrm{H}_{10} \mathrm{O}_{4} & 675,0 & 100,5 \\ \text { Valeriansäure, fest } & \mathrm{C}_{5} \mathrm{H}_{10} \mathrm{O}_{2} & 671,8 & 100 \\ \text { Isopropylmalonsäure } & \mathrm{C}_{6} \mathrm{H}_{10} \mathrm{O}_{4} & 675,2 & 100,5 \\ \text { Valeriansäure, fest } & \mathrm{C}_{5} \mathrm{H}_{10} \mathrm{O}_{2} & 671,8 & 100 \\ \text { Sym. Dimethylbernsteins. } & \mathrm{C}_{6} \mathrm{H}_{10} \mathrm{O}_{4} & 671,0 & 99,9 \\ \text { Valeriansäure, fest } & \mathrm{C}_{51} \mathrm{I}_{10} \mathrm{O}_{2} & 671,8 & 100 \\ \text { Unsym.Dimethylberusteins. } \mathrm{C}_{6} \mathrm{H}_{10} \mathrm{O}_{4} & 671,7 & 100 \\ \text { Valeriansäure, fest } & \mathrm{C}_{5} \mathrm{H}_{10} \mathrm{O}_{2} & 671,8 & 100 \\ \text { Aethylbernsteinsäure } & \mathrm{C}_{8} \mathrm{H}_{10} \mathrm{O}_{4} & 672,2 & 100,1 \\ \text { Valeriansäure, fest } & \mathrm{C}_{5} \mathrm{H}_{10} \mathrm{O}_{2} & 671,8 & 100 \\ \text { Methylglutarsäure } & \mathrm{C}_{6} \mathrm{H}_{10} \mathrm{O}_{4} & 670,8 & 99,8 \\ \text { Capronsäure, fest } & \mathrm{C}_{6} \mathrm{H}_{12} \mathrm{O}_{2} & 825,6 & 100 \\ \text { Pimelinsäure } & \mathrm{O}_{7} \mathrm{H}_{12} \mathrm{O}_{4} & 829,6 & 100,5 \\ \text { Oenanthsäure, fest } & \mathrm{C}_{7} \mathrm{H}_{14} \mathrm{O}_{2} & 979,4 & 100 \\ \text { Korksäure } & \mathrm{C}_{5} \mathrm{H}_{14} \mathrm{O}_{4} & 985,6 & 100,6 \\ \text { Caprylsäure, fest } & \mathrm{C}_{8} \mathrm{H}_{10} \mathrm{O}_{2} & 1133,1 & 100 \\ \text { Azelaïnsäure } & \mathrm{C}_{9} \mathrm{H}_{10} \mathrm{O}_{4} & 1141,3 & 100,7 \\ \text { Pelargonsäure, fest } & \mathrm{C}_{9} \mathrm{H}_{18} \mathrm{O}_{2} & 1287,2 & 100 \\ \text { Sebacinsäure } & \mathrm{C}_{10} \mathrm{H}_{18} \mathrm{O}_{4} & 1296,8 & 100,7 \\ & & & \end{array}$

Die einzige Süure, welche eine nennenswerthe Ausnahme von der allgemeinen Regel macht, ist die Bernsteinsäure; ihre Isomere, die Isobernsteinsäure, ist aber in ihrem Wärmewerthe der ihr entsprechenden einbasischen Säure völlig gleich.

4. Beziehungen der Fumar- und Maleïnsäure zu deı Bernsteinsäuren.

Es ist oben schon darauf hingewiesen, dass die Fumarsäure und Malë̈nsäure in ihren Wärmewerthen viel grössere Differenzen zeigen, als ihrer Constitution nach anzunehmen sein würde. Gauz analoge Differenzen finden wir bei den beiden Bernsteinsäuren:

$$
\begin{aligned}
& \text { Fumarsüure . . . . . } 320,1 \mathrm{Cal} . \\
& \text { Maleïnsäure . . . . . } 326,3 \text { " } \\
& \text { Bernsteinsäıre . . . . } 356,8 \text { Cal. } \\
& \text { Methylmalonsäure . . . } 364,8 \text { " }
\end{aligned}
$$


Wärmewerth der Säuren der 0xalsäure-Reihe etc. 225

Vergleichen wir die Wärmewerthe dieser beiden Säuregruppen:

$$
\begin{aligned}
& \text { Differenz: } \\
& \left.\begin{array}{lllll}
\text { Bernsteinsäure } & . & . & 356,8 \text { Cal. } \\
\text { Fumarsäure . . } & 320,1 \quad,
\end{array}\right\} 36,1 \text { Cal. } \\
& \left.\begin{array}{lll}
\text { Isobernsteinsäure . } & 364,8 \mathrm{Cal} \\
\text { Maleïnsäure . . . } & 326,3, \ldots
\end{array}\right\} 33,5 \mathrm{Cal} \text {. }
\end{aligned}
$$

so stehen die sich ergebenden Differenzen in völligem Einklange mit dem von Horstmann ${ }^{1}$ ) angegebenen Werthe. Nach Horstmann's Ermittelungen findet jedesmal eine Verringe. rung der Verbrennungswärme um 36 Cal. statt, wenn aus einem Kohlenwasserstoffe 2 Atome Wasserstoff austreten, und dadurch eine einfache Kohlenstoffbindung in eine doppelte verwandelt wird. Hervorgehoben muss jedoch werden, dass wir für einen analogen Vorgang: die Umwandlung der Hydrozimmtsäure in Zimmtsäure (Abh. XVIII $\left.{ }^{2}\right)$ ), eine etwas abweichende Zahl, nämlich 43,2 Cal., gefunden haben.

Leipzig, im Juli 1889.

In folgenden Tabellen sind die Einzelbeobachtungen, aus welchen die Zahlenwerthe abgeleitet sind, zusammengestellt. Die Verbrennungen geschahen immer in der'Bombe, unter einem Drucke von 24 Atmosphären. Bei den meisten Süuren erwies sich der Zusatz einer, meist minimalen Menge einer Zündmasse zur Einleitung der Verbrenıung als vortheilhaft. Als solche diente, überall wo nichts anderes bemerkt ist. Naphtalin, von welchem ein gewogener Krystall auf die Pastille gelegt wurde. Der Wärmewerth des Naphtalins ist, nach Abh. XVII ${ }^{3}$, mit 9628,3 cal. pro Gramm in Rechunng gestellt. In einzelnen wenigen Fällen ist Kerzenstearinsäure als Zündmasse benutzt. Da wo dieses geschehen ist, ist dieser Zusatz mit St. bezeichnet und dafür die in Abh. XVIII') ermittelte Zahl von 9374,1 cal. pro Gramm angenommen.

1) Ber. 21, 2217.

9) Dies. Journ. [2] 40, 150 .

3) Das. 40, 90. 1) Das. 40, 142 .

Jounal f. prakt. Chemie [2] Bd. 10. 
226 Stohmanu, Kleber u. Langhein: Ueber den

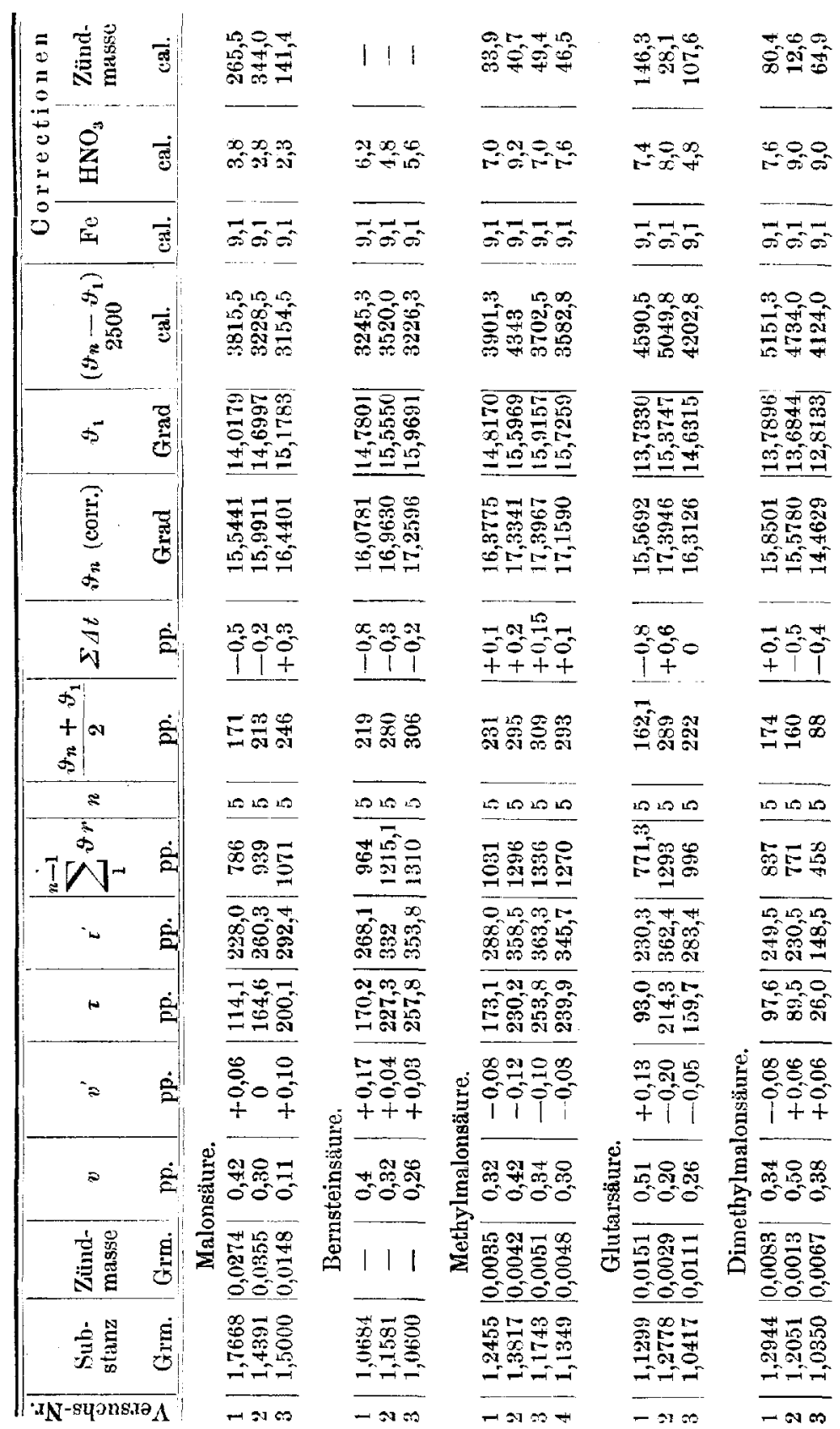


r. $0 \operatorname{los} 20$ $\sin _{x \rightarrow 0} \min ^{2}$

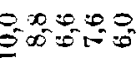

$\sin =$

$120, \log x$

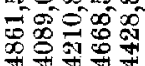

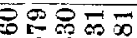

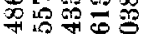

$\pm 0150$

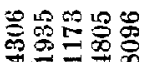

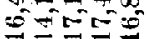

$\int_{0}^{1} \int_{0} \frac{i}{2}$

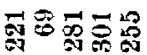

1020101040

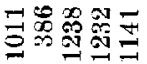

$\infty \infty+\infty$

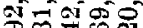

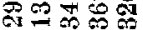

स- $=-1000$

車

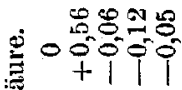

형

రీ

군

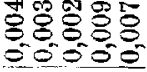

국도용

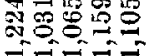

$-\alpha \cos$
0.0202

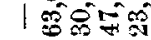

0100000

$-1-1-1-1$

कीक्षकी

$2100=000$

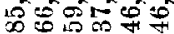

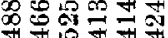

म워

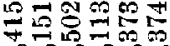

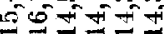

농

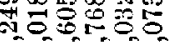

100000

$\cos x-1020$

$0-5000$

\section{$+t+1+$}

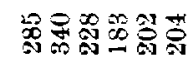

10 10 102010

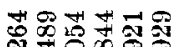

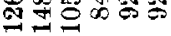

0.001024

बींगु की की

$\infty+\sigma x_{n} \omega$

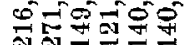

焉

능ㅇㅇㅇㅇㅇ

हीं

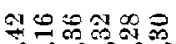
की

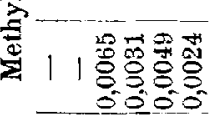

우우음

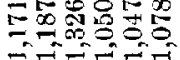

$-2 \pi+100$ $\infty 0_{0} 0$ एक ली

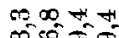
की

$\infty$
0,00

का ल

$\infty 0 \infty 0$ $0 x=\infty$

$\tan =0$

000

क्षेत

12040

ํํㅇํำ

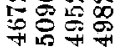

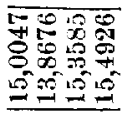

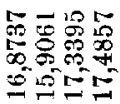

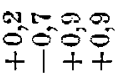

苗是总尔

$\cos 20100$

10

is os

की 1500

$\cos 00$

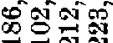

금

- 으용

की०0

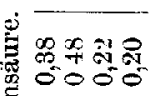
ह

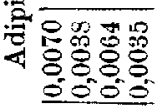

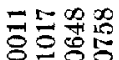

8085

$-\infty-\pi$

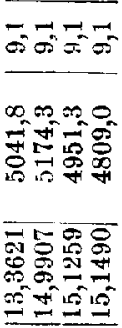

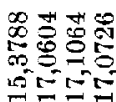

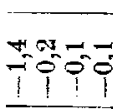

\section{垫器是}

-

………

$\forall 0$

三嫼

on $0-\infty$

-

$-0,0410$

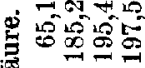

总

果苛

की

焉

oces 0 50

莪

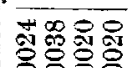

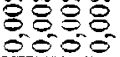

이요요

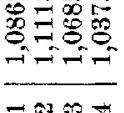

- 01 को
9015 $+1+$

m

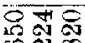
o. 15 15 15

200 on $5 x-5$

- $-\frac{1}{10}$ $\mathbb{*} 9$ 페 r. $0+\infty$ 45015 以

ن ड़ी

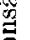

$C_{0}^{\infty}$ ठ0́ 象我 m

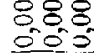
읭ㅇㅇ

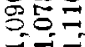

$-030$ 
228 Stohmann, Kleber u. Langbein: Leber den

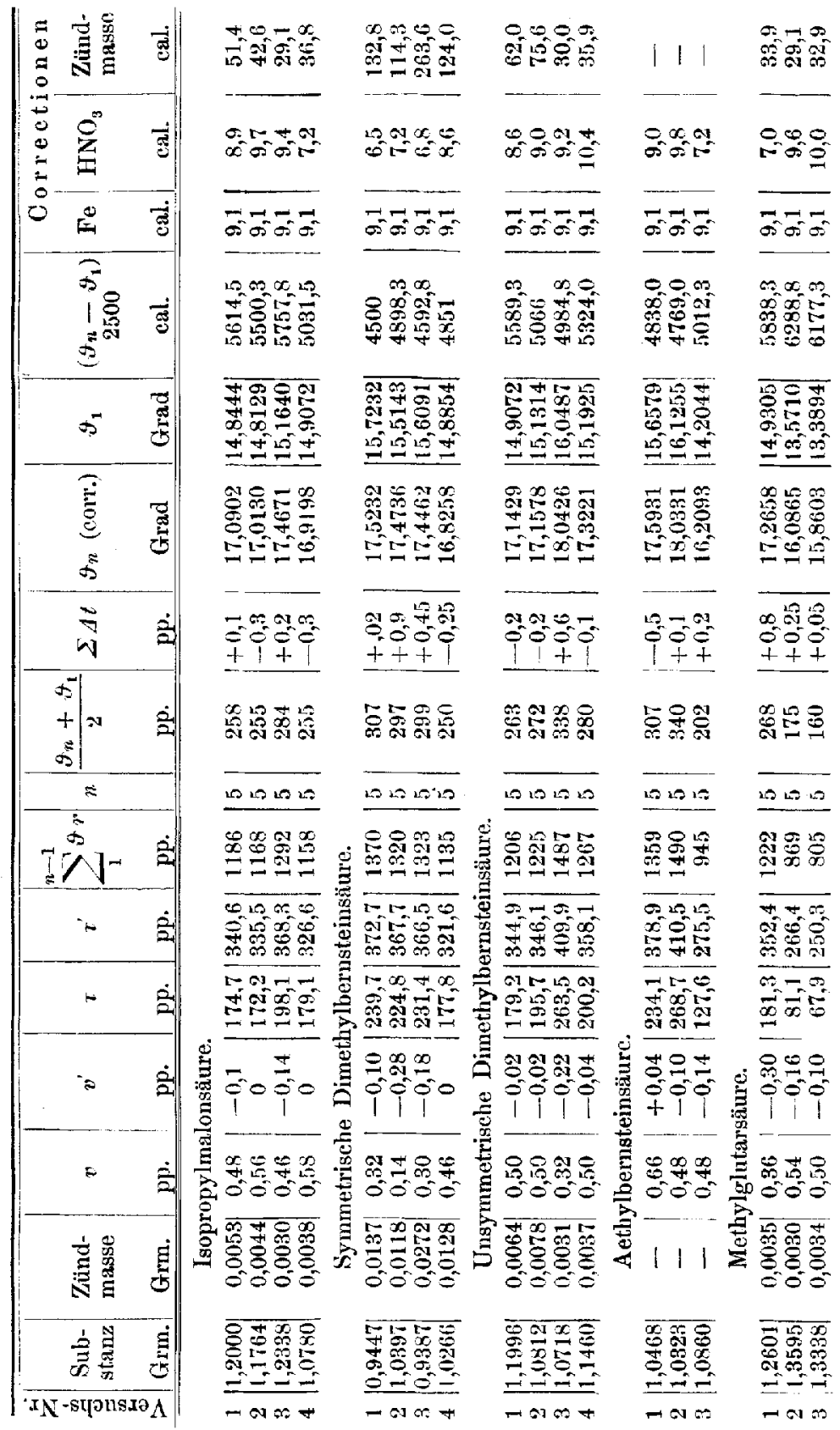


Wärmewerth der Säuren der 0xalsäure-Reihe etc. 229

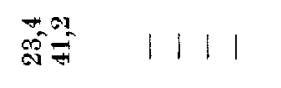

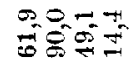

111

1.11

111

퐁요

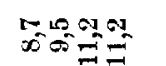

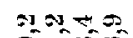

$\operatorname{lin} 20$

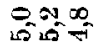

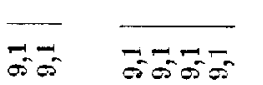

$-$

se=

काषिक

$\Rightarrow=-1$

-

$5=-5050$

$\Rightarrow=5$

$=-2=0$

000

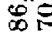

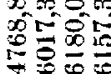

10,200

$200 \quad x+\infty m$

$\infty \infty$ - is 0

का

of

tes

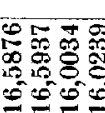

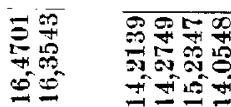

$\rightarrow-1$

$=\sigma \infty$

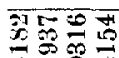

示果采

İ士

蟮

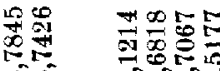

$\infty \infty \quad+000$

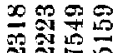

歌的

$\sin ^{\infty} x^{-1}$

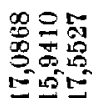

舟紧是是

B⿹

$15+0,5$

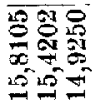

$\varphi$

to

$x_{-10}=0$

की

021

$-1$

50-

为漹

다이

$1=0$

000
$+1+$

+20

$\overbrace{0}^{\infty}$

10

15

8

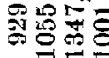

$\infty$

$\cos ^{10}$

Pnt=o

जी

In $\quad+\infty \infty 0$

कीज

거목

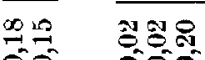

है

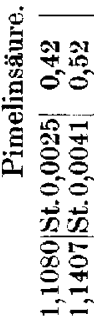

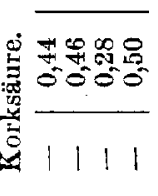

$+1++$

$0 \mathrm{nt}$

$1+1$

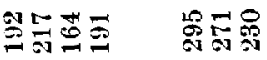

105020

70

20201010

$\cos 20$

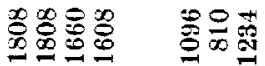

10000

웡응

$=\cos \infty$

क्षे

स $1=\infty$

बीं

\%

$0 \mathrm{st} t=\mathrm{cos}$

$1-2,01$

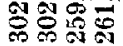

大

$00=0$

की

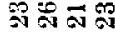

$01-000$

$x= \pm 0$

퍼듬펌

느응 잉

बิ

용워용

की 00

$0-10$

تึ

ल

L 0.5

충

000

눙요

०ิ00

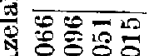

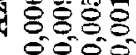
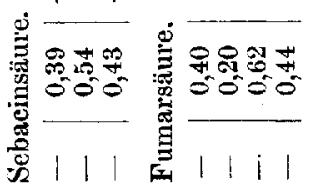

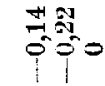

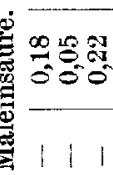

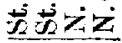

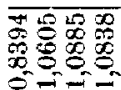

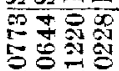

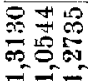

$x=0$
000
$0=0$

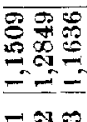

